\title{
ATOMIC SUBSPACES FOR OPERATORS
}

\author{
A. BHANDARI AND S. MUKHERJEE
}

\begin{abstract}
This paper introduces the concept of atomic subspaces with respect to a bounded linear operator. Atomic subspaces generalize fusion frames and this generalization leads to the notion of $K$-fusion frames. Characterizations of $K$-fusion frames are discussed. Various properties of $K$-fusion frames, for example, direct sum, intersection, are studied.
\end{abstract}

\section{INTRODUCTION}

Notion of Hilbert space frames was first introduced by Duffin and Schaeffer 6 in 1952 to reconstruct signals. Much later in the year 1986, the fundamental concept of frames and their significance in signal processing, image processing and data processing were presented by Daubechies, Grossman and Meyer [4. Frame theory plays an important role in various fields and have been widely applied in signal processing [8, sampling theory [7, coding and communications ([15], [11]) and so on.

It is a well-known fact that every element in a separable Hilbert space $\mathcal{H}$ can be explicitly represented as a linear combination of an orthonormal basis in $\mathcal{H}$ with the help of Fourier coefficients. But if one of the basis elements, for some reason, is removed, the explicit representation may not hold. Primarily due to this reason an overcomplete system was introduced which satisfies the explicit representation but more flexible when $f \in \mathcal{H}$ is to be reconstructed. Such an overcomplete system is called a "Frame".

L. Găvruţa in [9] was first to introduce the notion of $K$-frames to study the nature of atomic systems for a separable Hilbert space $\mathcal{H}$ with respect to a bounded linear operator $K$ on $\mathcal{H}$. In [10, Gãvruţa further studied atomic systems for operators in reproducing kernel Hilbert spaces, especially Bergman and Fock spaces. It is well-known fact that $K$-frames are more general than the classical frames and due to higher generality of $K$-frames, many properties of frames may not hold for $K$ frames.

In the 21st century scientists introduced fusion frames to handle massive amount of data to obtain mathematical framework to model and analyze such problems, which are otherwise almost impossible to handle. Moreover fusion frames are also significantly important mathematical gadget for theory oriented mathematical problems in frame theory. The notion of fusion frames (or frames of subspaces) was first introduced by Casazza et. al. (see [1, [2]). There are so many applications of fusion frames like coding theory, compressed sensing, data processing and so on. A fusion frame is a frame-like collection of closed subspaces in a Hilbert space.

2010 Mathematics Subject Classification. 42C15, 46C15.

Key words and phrases. Atomic subspaces, Frames, $K$-fusion frames.

Second author is supported by NIT Meghalaya Start-up Grant Project. 
In frame theory, amplitudes of projection vectors onto frame elements are used to represent signals whereas in the fusion frame theory, signals are represented by its projection vectors onto fusion frame subspaces. Also more specifically we may acquire that fusion frames are the generalization of conventional classical frames and special cases of $g$-frames in the field of frame theory.

This paper presents notion of atomic subspaces with respect to a bounded linear operator on a separable Hilbert space which leads to the concept of $K$-fusion frames, a generalization of fusion frames. This also generalize some results of $[9]$.

The paper is organized as follows. In Section 2, we recall basic definitions and results related to frames, $K$-frames and fusion frames. Atomic subspaces and $K$ fusion frames are introduced and discussed in Section 3. Finally in Section 4 we characterize $K$-fusion frames and establish various properties of the same.

Throughout the paper, $\mathcal{H}$ is a separable Hilbert space. We denote by $\mathcal{L}\left(\mathcal{H}_{1}, \mathcal{H}_{2}\right)$ the space of all bounded linear operators from $\mathcal{H}_{1}$ into $\mathcal{H}_{2}$. For $T \in \mathcal{L}(\mathcal{H})$, we denote $D(T), N(T)$ and $R(T)$ for domain, null space and range of $T$, respectively. We consider the index set $I$ to be finite or countable.

\section{Preliminaries}

In this section we recall basic definitions and results needed in this paper. We refer to the book by Ole Christensen $[3$ for an introduction to frame theory.

2.1. Frame. A collection $\left\{f_{i}\right\}_{i \in I}$ in $\mathcal{H}$ is called a frame if there exist constants $A, B>0$ such that

$$
A\|f\|^{2} \leq \sum_{i \in I}\left|\left\langle f, f_{i}\right\rangle\right|^{2} \leq B\|f\|^{2},
$$

for all $f \in \mathcal{H}$. The numbers $A, B$ are called frame bounds. The supremum over all $A$ 's and infimum over all $B$ 's satisfying above inequality are called the optimal frame bounds. The frame is called a tight frame if $A=B$ and if $A=B=1$ it is called a Parseval frame. The frame is called exact if it ceases to be a frame whenever any single element is removed from the collection. If a collection satisfies only the right inequality in (11), it is called a Bessel sequence.

Given a frame $\left\{f_{i}\right\}_{i \in I}$ of $\mathcal{H}$. The pre-frame operator or synthesis operator is a bounded linear operator $T: l^{2}(I) \rightarrow \mathcal{H}$ and is defined by $T\left\{c_{i}\right\}=\sum_{i \in I} c_{i} f_{i}$. The

adjoint of $T, T^{*}: \mathcal{H} \rightarrow l^{2}(I)$, given by $T^{*} f=\left\{\left\langle f, f_{i}\right\rangle\right\}$, is called the analysis operator. The frame operator, $S$, is obtained by composing $T$ with $T^{*}, S=T T^{*}$. That is, $S: \mathcal{H} \rightarrow \mathcal{H}$ such that

$$
S f=T T^{*} f=\sum_{i \in I}\left\langle f, f_{i}\right\rangle f_{i} .
$$

The frame operator is bounded, positive, self adjoint and invertible.

Reconstruction formula: Every element in $\mathcal{H}$ can be represented using frame elements as follows:

$$
f=\sum_{i \in I}\left\langle f, S^{-1} f_{i}\right\rangle f_{i}=\sum_{i \in I}\left\langle f, f_{i}\right\rangle S^{-1} f_{i}
$$

Since the frame elements are not necessarily linearly independent, this representation is not unique, in general. 
2.2. $K$-Frame. Let $K \in \mathcal{L}(\mathcal{H})$, then a sequence $\left\{f_{i}\right\}_{i \in I}$ in $\mathcal{H}$ is called a $K$-frame for $\mathcal{H}$ if there exist positive constants $A, B$ such that

$$
A\left\|K^{*} f\right\|^{2} \leq \sum_{i \in I}\left|\left\langle f, f_{i}\right\rangle\right|^{2} \leq B\|f\|^{2},
$$

for all $f \in \mathcal{H}$ and the above sequence is said to be a tight $K$-frame if

$$
A\left\|K^{*} f\right\|^{2}=\sum_{i \in I}\left|\left\langle f, f_{i}\right\rangle\right|^{2},
$$

for all $f \in \mathcal{H}$.

2.3. Fusion Frame. Given a Hilbert space $\mathcal{H}$, consider a collection of closed subspaces $\left\{\mathcal{W}_{i}\right\}_{i \in I}$ of $\mathcal{H}$ and a collection of positive weights $\left\{v_{i}\right\}_{i \in I}$. A family of weighted closed subspaces $(\mathcal{W}, v)=\left\{\left(\mathcal{W}_{i}, v_{i}\right): i \in I\right\}$ is called a fusion frame for $\mathcal{H}$, if there exist constants $0<A \leq B<\infty$ satisfying

$$
A\|f\|^{2} \leq \sum_{i \in I} v_{i}^{2}\left\|P_{\mathcal{W}_{i}} f\right\|^{2} \leq B\|f\|^{2},
$$

where $P_{\mathcal{W}_{i}}$ is the orthogonal projection from $\mathcal{H}$ onto $\mathcal{W}_{i}$. The constants $A$ and $B$ are called fusion frame bounds. If $A=B$ then the fusion frame is called a tight fusion frame, if $A=B=1$ then it is called a Parseval fusion frame and the fusion frame is called orthonormal if $\mathcal{H}=\bigoplus \mathcal{W}_{i}$. If $v_{i}=v, \forall i \in I$, it is called $v$-uniform fusion frame. A collection of closed subspaces, satisfying only the right inequality in 5, is called a fusion Bessel sequence.

For a family of closed subspaces, $\left\{\mathcal{W}_{i}\right\}_{i \in I}$, of $\mathcal{H}$, the corresponding $l^{2}$ space is defined by $\left(\sum_{i \in I} \oplus \mathcal{W}_{i}\right)_{l^{2}}=\left\{\left\{f_{i}\right\}_{i \in I}: f_{i} \in \mathcal{W}_{i}, \sum_{i \in I}\left\|f_{i}\right\|^{2}<\infty\right\}$ with inner product is given by $\left\langle\left\{f_{i}\right\},\left\{g_{i}\right\}\right\rangle=\sum_{i \in I}\left\langle f_{i}, g_{i}\right\rangle_{\mathcal{H}}$.

Let $\left\{\left(\mathcal{W}_{i}, v_{i}\right)\right\}_{i \in I}$ be a fusion frame. Then the synthesis operator $T_{\mathcal{W}}:\left(\sum_{i \in I} \oplus \mathcal{W}_{i}\right)_{l^{2}} \rightarrow$ $\mathcal{H}$ is defined as $T_{\mathcal{W}}(f)=\sum_{i \in I} v_{i} f_{i}$ for all $f=\left\{f_{i}\right\}_{i \in I} \in\left(\sum_{i \in I} \oplus \mathcal{W}_{i}\right)_{l^{2}}$ and the analysis operator $T_{\mathcal{W}}^{*}: \mathcal{H} \rightarrow\left(\sum_{i \in I} \oplus \mathcal{W}_{i}\right)_{l^{2}}$ is defined as $T_{\mathcal{W}}^{*}(f)=\left\{v_{i} P_{\mathcal{W}_{i}}(f)\right\}_{i \in I}$. It is well-known that (see [1]) the synthesis operator $T_{\mathcal{W}}$ of a fusion frame is bounded, linear and onto, whereas the corresponding analysis operator $T_{\mathcal{W}}^{*}$ is (possibly into) an isomorphism. Corresponding fusion frame operator is defined as $S_{\mathcal{W}}(f)=T_{\mathcal{W}} T_{\mathcal{W}}^{*}(f)=\sum_{i \in I} v_{i}^{2} P_{\mathcal{W}_{i}}(f) . S_{\mathcal{W}}$ is bounded, positive, self adjoint and invertible.

Reconstruction formula: Any signal $f \in \mathcal{H}$ can be expressed by its fusion frame measurements $\left\{v_{i} P_{\mathcal{W}_{i}} f\right\}_{i \in I}$ as

$$
f=\sum_{i \in I} v_{i} S_{\mathcal{W}}^{-1}\left(v_{i} P_{\mathcal{W}_{i}} f\right)
$$

Orthonormal basis in $\left(\sum_{i \in I} \bigoplus \mathcal{W}_{i}\right)_{l^{2}}$ : Consider a family of closed subspaces $\left\{\mathcal{W}_{i}\right\}_{i \in I}$ of $\mathcal{H}$. Let $\mathcal{U}=\left\{u_{1}, u_{2}, \ldots.\right\}$ be an orthonormal basis for $\mathcal{H}$ and consider a family of sets $\left\{J_{i}\right\}_{i \in I}$ such that $J_{i}=\mathcal{W}_{i} \cap \mathcal{U}$ and denote the cardinality of $J_{k}$ by $\left|J_{k}\right|$. For each $k \in I$, define $I$-tuples, $e_{k}^{l_{k}}=\left(\delta_{i}^{k} J_{k}\left(l_{k}\right)\right)_{i \in I}, l_{k}=1,2, \ldots,\left|J_{k}\right|$, where $J_{k}\left(l_{k}\right)$ is the $l_{k}$-th element of $J_{k}$. It is easy to verify that the collection $\left\{e_{i}^{l_{i}}\right\}_{i \in I}$ is countable and forms an orthonormal basis for $\left(\sum_{i \in I} \oplus \mathcal{W}_{i}\right)_{l^{2}}$.

We recall Douglas' factorization theorem (see [5]) which is required to present few results.

Theorem 2.1. (Douglas' factorization theorem) Let $\mathcal{H}_{1}, \mathcal{H}_{2}$, and $\mathcal{H}$ be Hilbert spaces and $S \in \mathcal{L}\left(\mathcal{H}_{1}, \mathcal{H}\right), T \in \mathcal{L}\left(\mathcal{H}_{2}, \mathcal{H}\right)$. Then the following are equivalent: 
(1) $R(S) \subseteq R(T)$.

(2) $S S^{*} \leq \alpha T T^{*}$ for some $\alpha>0$.

(3) $S=T L$ for some $L \in \mathcal{L}\left(\mathcal{H}_{1}, \mathcal{H}_{2}\right)$.

\section{Atomic subspaces}

We define atomic subspace of $\mathcal{H}$ with respect to a bounded linear operator.

Definition 3.1. Let $K \in \mathcal{L}(\mathcal{H})$ and consider a family of closed subspaces $\left\{\mathcal{W}_{i}\right\}_{i \in I}$ and a family of positive weights $\left\{v_{i}\right\}_{i \in I}$. Then $\left\{\left(\mathcal{W}_{i}, v_{i}\right)\right\}_{i \in I}$ is said to be an atomic subspace of $\mathcal{H}$ with respect to $K$ if the following conditions hold:

(a) $\sum_{i \in I} v_{i} f_{i}$ is convergent for all $\left\{f_{i}\right\}_{i \in I} \in\left(\sum_{i \in I} \oplus \mathcal{W}_{i}\right)_{l^{2}}$.

(b) For every $f \in \mathcal{H}$, there exist $\left\{f_{i}\right\}_{i \in I} \in\left(\sum_{i \in I} \oplus \mathcal{W}_{i}\right)_{l^{2}}$ such that $K f=$ $\sum_{i \in I} v_{i} f_{i}$ and $\left\|\left\{f_{i}\right\}\right\|_{\left(\sum_{i \in I} \oplus \mathcal{W}_{i}\right)_{l^{2}}} \leq C\|f\|_{\mathcal{H}}$ for some $C>0$.

Remark 3.2. Condition (a) in Definition 3.1 is equivalent to say that $\left\{\left(\mathcal{W}_{i}, v_{i}\right)\right\}_{i \in I}$ is a fusion Bessel sequence.

In the following we present the existence theorem of atomic subspaces.

Theorem 3.3. A separable Hilbert space has an atomic subspace with respect to every bounded linear operator.

Proof. Let $K \in \mathcal{L}(\mathcal{H})$ and consider $\left\{e_{n}\right\}_{n \in \mathbb{N}}$ as an orthonormal basis for $\mathcal{H}$. Define $\mathcal{U}_{n}=\operatorname{span}\left\{e_{n}\right\}$ and $\mathcal{W}_{n}=K\left(\mathcal{U}_{n}\right)$ for $n \in \mathbb{N}$. Then $\left\{\mathcal{U}_{n}\right\},\left\{\mathcal{W}_{n}\right\}$ form sequences of closed subspaces of $\mathcal{H}$. Also define $v_{n}=\left\|K e_{n}\right\|, n \in \mathbb{N}$. We claim that $\left\{\left(\mathcal{W}_{n}, v_{n}\right)\right\}_{n}$ forms an atomic subspace of $\mathcal{H}$ with respect to $K$.

To prove this first note that for every $f \in \mathcal{H}, P_{\mathcal{W}_{n}} f=\frac{\left\langle f, K e_{n}\right\rangle}{v_{n}^{2}} K e_{n}$. Hence we have

$$
\sum_{n \in \mathbb{N}} v_{n}^{2}\left\|P_{\mathcal{W}_{n}} f\right\|^{2}=\sum_{n \in \mathbb{N}}\left|\left\langle f, K e_{n}\right\rangle\right|^{2}=\left\|K^{*} f\right\|^{2} \leq\|K\|^{2}\|f\|^{2} .
$$

This shows that $\left\{\left(\mathcal{W}_{n}, v_{n}\right)\right\}_{n}$ is a fusion Bessel sequence.

Again for all $f \in \mathcal{H}, f=\sum_{n \in \mathbb{N}}\left\langle f, e_{n}\right\rangle e_{n}$ and therefore $K f=\sum_{n \in \mathbb{N}}\left\langle f, e_{n}\right\rangle K e_{n}=$ $\sum_{n \in \mathbb{N}} v_{n} f_{n}$, where $f_{n}=\frac{\left\langle f, e_{n}\right\rangle}{\left\|K e_{n}\right\|} K e_{n} \in \mathcal{W}_{n}$. Thus we have

$$
\left\|\left\{f_{n}\right\}\right\|_{\left(\sum_{n \in \mathbb{N}} \oplus \mathcal{W}_{n}\right)_{l^{2}}}^{2}=\sum_{n \in \mathbb{N}}\left\|f_{n}\right\|^{2}=\sum_{n \in \mathbb{N}}\left|\left\langle f, e_{n}\right\rangle\right|^{2}=\|f\|^{2} .
$$

The notion of atomic subspaces has revived to produce generalization of family of local atoms or atomic systems for a bounded, linear operator. The following theorem provides a characterization of atomic subspaces.

Theorem 3.4. Let $\mathcal{H}$ be a Hilbert space. Assume that $\left\{\mathcal{W}_{i}\right\}_{i \in I}$ be a family of closed subspaces of $\mathcal{H}$ and $\left\{v_{i}\right\}_{i \in I}$ be a family of positive weights. Then the following statements are equivalent:

(1) $\left\{\left(\mathcal{W}_{i}, v_{i}\right)\right\}_{i \in I}$ is an atomic subspace of $\mathcal{H}$ with respect to $K$.

(2) There exist $A, B>0$ such that for all $f \in \mathcal{H}$,

$$
A\left\|K^{*} f\right\|^{2} \leq \sum_{i \in I} v_{i}^{2}\left\|P_{\mathcal{W}_{i}} f\right\|^{2} \leq B\|f\|^{2} .
$$


Proof. Suppose $\left\{\left(\mathcal{W}_{i}, v_{i}\right)\right\}_{i \in I}$ is an atomic subspace of $\mathcal{H}$ with respect to $K$. It is sufficient to show that there exists a constant $A>0$ such that $\sum_{i \in I} v_{i}^{2}\left\|P_{\mathcal{W}_{i}} f\right\|^{2} \geq$ $A\left\|K^{*} f\right\|^{2}$ for all $f \in \mathcal{H}$. But since $\left\|T_{\mathcal{W}}^{*} f\right\|^{2}=\sum_{i \in I} v_{i}^{2}\left\|P_{\mathcal{W}_{i}} f\right\|^{2}$, where $T_{\mathcal{W}}$ is the corresponding synthesis operator, this is equivalent to show that $T_{\mathcal{W}} T_{\mathcal{W}}^{*} \geq A K K^{*}$. Now since $T_{\mathcal{W}}$ is bounded, linear, onto [1, $R\left(T_{\mathcal{W}}\right) \supseteq R(K)$. Therefore by using Theorem 2.1 we get the desired result.

Conversely, suppose that the inequality in 2 is true. Then the right inequality asserts that $\left\{\left(\mathcal{W}_{i}, v_{i}\right)\right\}_{i \in I}$ is a fusion Bessel sequence. Now the left inequality gives $A K K^{*} \leq T_{\mathcal{W}} T_{\mathcal{W}}^{*}$. Then using Theorem 2.1 there exists a bounded linear operator $L \in \mathcal{L}\left(\mathcal{H},\left(\sum_{i \in I} \oplus \mathcal{W}_{i}\right)_{l^{2}}\right)$ such that $K=T_{\mathcal{W}} L$. For every $f \in \mathcal{H}$, define $L f=\left\{f_{i}\right\}_{i \in I}$. Therefore $K f=T_{\mathcal{W}}\left\{f_{i}\right\}_{i \in I}=\sum_{i \in I} v_{i} f_{i}$ and $\left\|\left\{f_{i}\right\}\right\|_{\left(\sum_{i \in I} \oplus \mathcal{W}_{i}\right)_{l^{2}}}=$

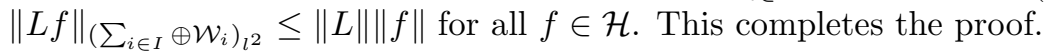

Corollary 3.5. Let $\left\{\left(\mathcal{W}_{i}, v_{i}\right)\right\}_{i \in I}$ be a fusion Bessel sequence in $\mathcal{H}$. Then $\left\{\left(\mathcal{W}_{i}, v_{i}\right)\right\}_{i \in I}$ is an atomic subspace of $\mathcal{H}$ with respect to the corresponding fusion frame operator $S_{\mathcal{W}} f=\sum_{i \in I} v_{i}^{2} P_{\mathcal{W}_{i}}(f)$, for all $f \in \mathcal{H}$.

Proof. Given that $\left\{\left(\mathcal{W}_{i}, v_{i}\right)\right\}_{i \in I}$ be a fusion Bessel sequence in $\mathcal{H}$. Then $\sum_{i \in I} v_{i}^{2}\left\|P_{\mathcal{W}_{i}} f\right\|^{2} \leq$ $B\|f\|^{2}$ for all $f \in \mathcal{H}$ and for some $B>0$. Now since $R\left(T_{\mathcal{W}}\right)=\mathcal{H}=R\left(S_{\mathcal{W}}\right)$, by using Douglas theorem (2.1), we have $A S_{\mathcal{W}} S_{\mathcal{W}}^{*} \leq T_{\mathcal{W}} T_{\mathcal{W}}^{*}$, for some $A>0$. Hence $A\left\|S_{\mathcal{W}}^{*} f\right\|^{2} \leq \sum_{i \in I} v_{i}^{2}\left\|P_{\mathcal{W}_{i}} f\right\|^{2}$ and hence the result follows from Theorem 3.4 .

Theorem 3.4 provides a generalization of fusion frames.

Definition 3.6. Given $\mathcal{H}$, a collection of closed subspaces $\left\{\mathcal{W}_{i}\right\}_{i \in I}$ of $\mathcal{H}$ with a collection of positive weights $\left\{v_{i}\right\}_{i \in I},\left\{\left(\mathcal{W}_{i}, v_{i}\right): i \in I\right\}$, is said to be a K-fusion frame for $\mathcal{H}$ with respect to $K \in \mathcal{L}(\mathcal{H})$ if there exist positive constants $A, B$ such that

$$
A\left\|K^{*} f\right\|^{2} \leq \sum_{i \in I} v_{i}^{2}\left\|P \mathcal{W}_{i} f\right\|^{2} \leq B\|f\|^{2} .
$$

In this context, we acknowledge that recently Liu and $\mathrm{Li}$ 13 introduced the concept of $K$-fusion frames. They studied $K$-fusion frames with unitary systems' structure and introduced the concept of $K$-fusion frame generators. The present work has been done almost simultaneously with the work of Liu and Li.

Here we recall the definition of Moore-Penrose pseudo inverse of an operator.

Definition 3.7. 12] Let $\mathcal{H}$ be a Hilbert space and suppose that $T \in \mathcal{L}(\mathcal{H})$ has closed range. Then there exists an operator $T^{\dagger} \in \mathcal{L}(\mathcal{H})$ for which

$$
N\left(T^{\dagger}\right)=N\left(T^{*}\right), R\left(T^{\dagger}\right)=R\left(T^{*}\right), T T^{\dagger}=P_{R(T)}, T^{\dagger} T=P_{R\left(T^{*}\right)} .
$$

$T^{\dagger}$ is called Moore-Penrose pseudo inverse of $T$ and is uniquely determined by the above mentioned properties. If $T$ is invertible, then $T^{-1}=T^{\dagger}$.

The following theorem provides a relation between fusion frames and $K$-fusion frames.

Theorem 3.8. Let $K \in \mathcal{L}(\mathcal{H})$. Then:

(a) Every fusion frame is a $K$-fusion frame.

(b) If $R(K)$ is closed, every $K$-fusion frame is a fusion frame for $R(K)$. 
Proof. (a) Let $\left\{\left(\mathcal{W}_{i}, v_{i}\right)\right\}_{i \in I}$ be a fusion frame for $\mathcal{H}$ with frame bounds $A, B$. Then for all $f \in \mathcal{H}$,

$$
\frac{A}{\|K\|^{2}}\left\|K^{*} f\right\|^{2} \leq A\|f\|^{2} \leq \sum_{i \in I} v_{i}^{2}\left\|P_{\mathcal{W}_{i}} f\right\|^{2} \leq B\|f\|^{2} .
$$

(b) Let $\left\{\left(\mathcal{W}_{i}, v_{i}\right)\right\}_{i \in I}$ be a $K$-fusion frame for $\mathcal{H}$ with frame bounds $A, B$. Then for all $f \in R(K)$,

$$
\frac{A}{\left\|K^{* \dagger}\right\|^{2}}\|f\|^{2} \leq A\left\|K^{*} f\right\|^{2} \leq \sum_{i \in I} v_{i}^{2}\left\|P_{\mathcal{W}_{i}} f\right\|^{2} \leq B\|f\|^{2} .
$$

\section{RESUlts}

In this section we discuss properties of atomic subspaces and characterize the same.

We recall the quotient of bounded operators (see [14]).

Definition 4.1. Let $A, B \in \mathcal{L}(\mathcal{H})$ with $N(B) \subset N(A)$. The quotient operator $T=[A / B]$ is a map from $R(B)$ to $R(A)$ defined by $B x \mapsto A x$.

It may be noted that $D(T)=R(B), R(T) \subset R(A)$ and $T B=A$.

Usefulness of Bessel sequence in frame theory and in general in mathematical analysis is well known. Similarly the concept of fusion Bessel sequence gives us so many spin-off results in fusion frame theory. In the following two theorems 44.2, 4.4) we present necessary and sufficient conditions for fusion Bessel sequence to be $K$-fusion frame.

Theorem 4.2. Let $\left\{\left(\mathcal{W}_{i}, v_{i}\right)\right\}_{i \in I}$ be a fusion Bessel sequence in $\mathcal{H}$ with corresponding fusion frame operator $S_{\mathcal{W}}$ and assume that $K \in \mathcal{L}(\mathcal{H})$. Then $\left\{\left(\mathcal{W}_{i}, v_{i}\right)\right\}_{i \in I}$ is a $K$-fusion frame if and only if the quotient operator $\left[K^{*} / S_{\mathcal{W}}^{1 / 2}\right]$ is bounded.

Proof. Let $\left\{\left(\mathcal{W}_{i}, v_{i}\right)\right\}_{i \in I}$ be a $K$-fusion frame. Then there is a constant $A_{1}>0$ such that

$$
A_{1}\left\|K^{*} f\right\|^{2} \leq \sum_{i \in I} v_{i}^{2}\left\|P_{\mathcal{W}_{i}} f\right\|^{2}=\left\langle S_{\mathcal{W}} f, f\right\rangle=\left\|S_{\mathcal{W}}^{1 / 2} f\right\|^{2},
$$

for all $f \in \mathcal{H}$. Now let us denote the quotient operator $\left[K^{*} / S_{\mathcal{W}}^{1 / 2}\right]$ by $T$. Then $T: R\left(S_{\mathcal{W}}^{1 / 2}\right) \rightarrow R\left(K^{*}\right)$ such that $T\left(S_{\mathcal{W}}^{1 / 2} f\right)=K^{*} f$ for all $f \in \mathcal{H}$. From 8 , it is clear that $N\left(S_{\mathcal{W}}^{1 / 2}\right) \subset N\left(K^{*}\right)$ and thus $T$ is well defined. Also $\left\|T\left(S_{\mathcal{W}}^{1 / 2} f\right)\right\|=\left\|K^{*} f\right\| \leq$ $\frac{1}{\sqrt{A_{1}}}\left\|S_{\mathcal{W}}^{1 / 2} f\right\|$ for all $f \in \mathcal{H}$ and hence $T$ is bounded.

Conversely, suppose that the quotient operator $\left[K^{*} / S_{\mathcal{W}}^{1 / 2}\right]$ is bounded. Then there exists a constant $A_{2}>0$ such that $\left\|K^{*} f\right\|^{2} \leq A_{2}\left\|S_{\mathcal{W}}^{1 / 2} f\right\|^{2}=A_{2}\left\langle S_{\mathcal{W}} f, f\right\rangle=$ $A_{2} \sum_{i \in I} v_{i}^{2}\left\|P_{\mathcal{W}_{i}} f\right\|^{2}$ for all $f \in \mathcal{H}$ and consequently $\left\{\left(\mathcal{W}_{i}, v_{i}\right)\right\}_{i \in I}$ forms a $K$-fusion frame for $\mathcal{H}$.

Corollary 4.3. Let $\mathcal{H}$ be a Hilbert space. Let $\left\{\left(\mathcal{W}_{i}, v_{i}\right)\right\}_{i \in I}$ be a fusion Bessel sequence in $\mathcal{H}$ with the fusion frame operator $\mathcal{S}_{\mathcal{W}}$. Then $\left\{\left(\mathcal{W}_{i}, v_{i}\right)\right\}_{i \in I}$ is a fusion frame if and only if $\mathcal{S}_{\mathcal{W}}$ is invertible and positive. 
Proof. One direction is obvious from the definition and the fact that $\left\langle S_{\mathcal{W}} f, f\right\rangle=$ $\sum_{i \in I} v_{i}^{2}\left\|P_{\mathcal{W}_{i}} f\right\|^{2}$.

Conversely, let us assume that $\mathcal{S}_{\mathcal{W}}$ is invertible and positive. Then the result follows from Theorem 4.2 with $K=I$.

Theorem 4.4. Let $\left\{\left(\mathcal{W}_{i}, v_{i}\right)\right\}_{i \in I}$ be a fusion Bessel sequence in $\mathcal{H}$ with fusion frame operator $S_{\mathcal{W}}$ and $K \in \mathcal{L}(\mathcal{H})$, then $\left\{\left(\mathcal{W}_{i}, v_{i}\right)\right\}_{i \in I}$ is a $K$-fusion frame for $\mathcal{H}$ if and only if there exists a positive constant $A$ such that $S_{\mathcal{W}} \geq A K K^{*}$.

Proof. The proof follows from the fact that $\left\langle S_{\mathcal{W}} f, f\right\rangle=\sum_{i \in I} v_{i}^{2}\left\|P_{\mathcal{W}_{i}} f\right\|^{2}$. See [16] for details.

Here we present a necessary and sufficient condition for a family of closed subspaces to be a $K$-fusion frame.

Theorem 4.5. Let $\mathcal{H}$ be a Hilbert space and $K \in \mathcal{L}(\mathcal{H})$. Assume that $\left\{\mathcal{W}_{i}\right\}_{i \in I}$ be a family of closed subspaces of $\mathcal{H}$ and $\left\{v_{i}\right\}_{i \in I}$ be a family of positive weights. Then $\left\{\left(\mathcal{W}_{i}, v_{i}\right)\right\}_{i \in I}$ is a $K$-fusion frame for $\mathcal{H}$ if and only if there exists a bounded, linear operator $L:\left(\sum_{i \in I} \oplus \mathcal{W}_{i}\right)_{l^{2}} \rightarrow \mathcal{H}$ such that $L e_{n}=\sum_{i \in I} v_{i} e_{n}^{i}$ and $R(K) \subseteq$ $R(L)$, where $\left\{e_{n}\right\}_{n=1}^{\infty}$ is an orthonormal basis in $\left(\sum_{i \in I} \oplus \mathcal{W}_{i}\right)_{l^{2}}$ and $e_{n}^{i}$ is the $i$-th component of $e_{n}$.

Proof. Let $\left\{\left(\mathcal{W}_{i}, v_{i}\right)\right\}_{i \in I}$ be a $K$-fusion frame. Then there exist positive constants $A$ and $B$ such that

$$
A\left\|K^{*} f\right\|^{2} \leq \sum_{i \in I} v_{i}^{2}\left\|P_{\mathcal{W}_{i}} f\right\|^{2} \leq B\|f\|^{2},
$$

for all $f \in \mathcal{H}$. Define $L^{*}: \mathcal{H} \rightarrow\left(\sum_{i \in I} \oplus \mathcal{W}_{i}\right)_{l^{2}}$ such that $L^{*} f=\left\{v_{i} P_{\mathcal{W}_{i}} f\right\}_{i \in I}$. Then $\left\|L^{*} f\right\|^{2}=\sum_{i \in I} v_{i}^{2}\left\|P_{\mathcal{W}_{i}} f\right\|^{2}$ for all $f \in \mathcal{H}$. Hence by the previous inequality we have $A\left\|K^{*} f\right\|^{2} \leq\left\|L^{*} f\right\|^{2}$ for all $f \in \mathcal{H}$ and therefore $A K K^{*} \leq L L^{*}$. Therefore by Theorem 2.1. $R(K) \subset R(L)$. Now $\left\langle f, L e_{n}\right\rangle_{\mathcal{H}}=\left\langle L^{*} f, e_{n}\right\rangle_{\left(\sum_{i \in I} \oplus \mathcal{W}_{i}\right)_{l^{2}}}=$ $\left\langle\left\{v_{i} P_{\mathcal{W}_{i}} f\right\}, e_{n}\right\rangle_{\left(\sum_{i \in I} \oplus \mathcal{W}_{i}\right)_{l^{2}}}=\sum_{i \in I}\left\langle v_{i} P_{\mathcal{W}_{i}} f, e_{n}^{i}\right\rangle_{\mathcal{H}}=\sum_{i \in I}\left\langle f, v_{i} e_{n}^{i}\right\rangle_{\mathcal{H}}$. Hence $L e_{n}=$ $\sum_{i \in I} v_{i} e_{n}^{i}$.

Conversely, suppose $L:\left(\sum_{i \in I} \oplus \mathcal{W}_{i}\right)_{l^{2}} \rightarrow \mathcal{H}$ such that $L e_{n}=\sum_{i \in I} v_{i} e_{n}^{i}$ and $R(K) \subset R(L)$. Then $L^{*} f=\left\{v_{i} P_{\mathcal{W}_{i}} f\right\}_{i \in I}$. Therefore $\sum_{i \in I} v_{i}^{2}\left\|P_{\mathcal{W}_{i}} f\right\|^{2}=\left\|L^{*} f\right\|^{2} \leq$ $\left\|L^{*}\right\|^{2}\|f\|^{2}$. Hence $\left\{\left(\mathcal{W}_{i}, v_{i}\right)\right\}_{i \in I}$ form a fusion Bessel sequence. Now since $R(K) \subseteq$ $R(L)$, again by Theorem 2.1, there exists a positive constant $A$ such that $A K K^{*} \leq$ $L L^{*}$ and hence $A\left\|K^{*} f\right\|^{2} \leq\left\|L^{*} f\right\|^{2}=\sum_{i \in I} v_{i}^{2}\left\|P_{\mathcal{W}_{i}} f\right\|^{2}$. Consequently $\left\{\left(\mathcal{W}_{i}, v_{i}\right)\right\}_{i \in I}$ is a $K$-fusion frame for $\mathcal{H}$.

Following two results show methods of construction of $K$-fusion frames from $K$-frames. Analogous results for fusion frames are discussed in [1].

Theorem 4.6. Let $\mathcal{H}$ be a Hilbert space, $K \in \mathcal{L}(\mathcal{H})$ and $\left\{f_{j}\right\}_{j \in J}$ be a $K$-frame for $\mathcal{H}$ with frame bounds $A$ and $B$. Assume that $\left\{J_{i}\right\}_{i \in I}$ is a partition of the index set $J$ and $\mathcal{W}_{i}$ is the closed linear span of $\left\{f_{j}\right\}_{j \in J_{i}}$ for all $i \in I$. Then for all $f \in \mathcal{H}$ we have $\frac{A}{B}\left\|K^{*} f\right\|^{2} \leq \sum_{i \in I}\left\|P_{\mathcal{W}_{i}} f\right\|^{2}$. Further if $|I|<\infty$ then $\left\{\mathcal{W}_{i}\right\}_{i \in I}$ is an 1-uniform $K$-fusion frame for $\mathcal{H}$.

Proof. Since $\left\{f_{j}\right\}_{j \in J}$ is a $K$-frame for $\mathcal{H}$ with bounds $A$ and $B$, we have

$$
A\left\|K^{*} f\right\|^{2} \leq \sum_{j \in J}\left|\left\langle f, f_{j}\right\rangle\right|^{2}=\sum_{i \in I} \sum_{j \in J_{i}}\left|\left\langle f, f_{j}\right\rangle\right|^{2}=\sum_{i \in I} \sum_{j \in J_{i}}\left|\left\langle P_{\mathcal{W}_{i}} f, f_{j}\right\rangle\right|^{2} \leq B\|f\|^{2},
$$


for all $f \in \mathcal{H}$. Now since every sub-collection of a Bessel sequence is also a Bessel, we have $\sum_{i \in I} \sum_{j \in J_{i}}\left|\left\langle P_{\mathcal{W}_{i}} f, f_{j}\right\rangle\right|^{2} \leq \sum_{i \in I} B\left\|P_{\mathcal{W}_{i}} f\right\|^{2}$. Hence we have $\frac{A}{B}\left\|K^{*} f\right\|^{2} \leq$ $\sum_{i \in I}\left\|P_{\mathcal{W}_{i}} f\right\|^{2}$, for all $f \in \mathcal{H}$.

Further, if $|I|<\infty$ then we have $\sum_{i \in I}\left\|P_{\mathcal{W}_{i}} f\right\|^{2} \leq|I|\|f\|^{2}$. Hence in this special case $\left\{\mathcal{W}_{i}\right\}_{i \in I}$ is always an 1-uniform $K$-fusion frame for $\mathcal{H}$.

Corollary 4.7. Let $\mathcal{H}$ be a Hilbert space and $K \in \mathcal{L}(\mathcal{H})$. Suppose $\left\{f_{j}\right\}_{j \in J}$ is a $K$-frame for $\mathcal{H}$. Assume that $J=J_{1} \cup J_{2} \cup \ldots \cup J_{n}$ be a finite partition of $J$ and $\left.\mathcal{W}_{i}=\overline{\operatorname{span}_{j \in J_{i}}\left\{f_{j}\right.}\right\}$. Then $\left\{\left(\mathcal{W}_{i}, v_{i}\right)\right\}_{i=1}^{n}$ forms a $K$-fusion frame for $\mathcal{H}$ for any collection of positive weights $\left\{v_{i}\right\}_{i=1}^{n}$.

Proof. Let $\left\{f_{j}\right\}_{j \in J}$ be a $K$-frame for $\mathcal{H}$ with frame bounds $A$ and $B$. Then using Theorem [4.6. $\left\{W_{i}\right\}$ forms an 1-uniform $K$-fusion frame with frame bounds $A / B$ and $n$. That is

$$
\frac{A}{B}\left\|K^{*} f\right\|^{2} \leq \sum_{i=1}^{n}\left\|P_{\mathcal{W}_{i}} f\right\|^{2} \leq n\|f\|^{2}
$$

for all $f \in \mathcal{H}$. Now considering $v=\min \left\{v_{i}: i=1,2, \cdots, n\right\}$ and $w=\max \left\{v_{i}: i=\right.$ $1,2, \cdots, n\}$, we have

$$
\frac{A v^{2}}{B}\left\|K^{*} f\right\|^{2} \leq \sum_{i=1}^{n} v_{i}^{2}\left\|P_{\mathcal{W}_{i}} f\right\|^{2} \leq n w^{2}\|f\|^{2},
$$

for all $f \in \mathcal{H}$. Hence proved.

Definition 4.8. Let $\left\{\mathcal{H}_{i}\right\}_{i \in I}$ be a non-overlapping family of Hilbert spaces. For each $i \in I$, let us assume that $T_{i}: \mathcal{H}_{i} \rightarrow \mathcal{H}_{i}$ be a bounded, linear operator on $\mathcal{H}_{i}$ such that the family $\left\{T_{i}\right\}_{i \in I}$ is uniformly bounded i.e. $\sup \left\{\left\|T_{i}\right\|: i \in I\right\}<\infty$. Then the direct sum operator of the uniformly bounded family $\left\{T_{i}\right\}_{i \in I}$ is the operator $\bigoplus_{i \in I} T_{i}: \bigoplus_{i \in I} \mathcal{H}_{i} \rightarrow \bigoplus_{i \in I} \mathcal{H}_{i}$ on the direct sum of the Hilbert spaces $\bigoplus_{i \in I} \mathcal{H}_{i}$ is defined as $\left(\bigoplus_{i \in I} T_{i}\right)(x)=\sum_{i \in I} T_{i} x_{i}$, where $x=\sum_{i \in I} x_{i}$ and $x_{i} \in \mathcal{H}_{i}$.

It is easy to check that $\bigoplus_{i \in I} T_{i}$ is well defined, bounded, linear operator, whose norm is given by $\left\|\bigoplus_{i \in I} T_{i}\right\|=\sup \left\{\left\|T_{i}\right\|: i \in I\right\}$.

In the following theorem we will show that direct sum of $K$-fusion frames is a $K$-fusion frame.

Theorem 4.9. Let $\left\{\left(\mathcal{W}_{i j}, v_{i}\right)\right\}_{i \in I}$ be a collection of $K_{j}$-fusion frames for $\mathcal{H}_{j}, j=$ $1,2, \cdots, m$ with $\mathcal{W}_{i j} \cap \mathcal{W}_{i k}=\phi$ for $j \neq k$. Then $\left\{\bigoplus_{j=1}^{m} \mathcal{W}_{i j}, v_{i}\right\}_{i \in I}$ is a $\bigoplus_{j=1}^{m} K_{j}$ fusion frame for the Hilbert space $\bigoplus_{j=1}^{m} H_{j}$.

Proof. It is sufficient to prove the result for $m=2$. Let $A_{j}$ and $B_{j}$ be frame bounds for the $K_{j}$-fusion frame $\left\{\left(\mathcal{W}_{i j}, v_{i}\right)\right\}_{i \in I} j=1,2$. Since $P_{\mathcal{W}_{i 1} \oplus \mathcal{W}_{i 2}}=P_{\mathcal{W}_{i 1}} \oplus P_{\mathcal{W}_{i 2}}$ 
$(i \in I)$, then for all $f \in \mathcal{H}_{1}$ and $g \in \mathcal{H}_{2}$ we have,

$$
\begin{aligned}
\min \left\{A_{1}, A_{2}\right\}\left\|\left(K_{1} \oplus K_{2}\right)^{*}(f \oplus g)\right\|^{2} & =\min \left\{A_{1}, A_{2}\right\}\left\|\left(K_{1}^{*} \oplus K_{2}^{*}\right)(f \oplus g)\right\|^{2} \\
& =\min \left\{A_{1}, A_{2}\right\}\left\|K_{1}^{*} f \oplus K_{2}^{*} g\right\|^{2} \\
& =\min \left\{A_{1}, A_{2}\right\}\left(\left\|K_{1}^{*} f\right\|^{2}+\left\|K_{2}^{*} g\right\|^{2}\right) \\
& \leq A_{1}\left\|K_{1}^{*} f\right\|^{2}+A_{2}\left\|K_{2}^{*} g\right\|^{2} \\
& \leq \sum_{i \in I} v_{i}^{2}\left\|P_{\mathcal{W}_{i 1}}(f)\right\|^{2}+\sum_{i \in I} v_{i}^{2}\left\|P_{\mathcal{W}_{i 2}}(g)\right\|^{2} \\
& \leq B_{1}\|f\|^{2}+B_{2}\|g\|^{2} \\
& \leq \max \left\{B_{1}, B_{2}\right\}\left(\|f\|^{2}+\|g\|^{2}\right) \\
& =\max \left\{B_{1}, B_{2}\right\}\|f \oplus g\|^{2} .
\end{aligned}
$$

Result follows from the fact that $\sum_{i \in I} v_{i}^{2}\left\|P_{\mathcal{W}_{i 1}}(f)\right\|^{2}+\sum_{i \in I} v_{i}^{2}\left\|P_{\mathcal{W}_{i 2}}(g)\right\|^{2}=\sum_{i \in I} v_{i}^{2} \| P_{\mathcal{W}_{i 1} \oplus \mathcal{W}_{i 2}}(f \oplus$ g) $\|^{2}$.

In the following result we will present some algebraic properties of $K$-fusion frame.

Theorem 4.10. Let $K_{j} \in \mathcal{L}(\mathcal{H})$ and $\left\{a_{j}\right\}$ be a finite collection of scalars for $j=1,2, \cdots, n$. Suppose $\left\{\left(\mathcal{W}_{i}, v_{i}\right)\right\}_{i \in I}$ is a $K_{j}$-fusion frame for $\mathcal{H}$, for all $j=$ $1,2, \cdots, n$. Then $\left\{\left(\mathcal{W}_{i}, v_{i}\right)\right\}_{i \in I}$ is also a $\sum_{j=1}^{n} a_{j} K_{j}$-fusion frame and $\prod_{j=1}^{n} K_{j}$ fusion frame for $\mathcal{H}$.

Proof. Since $\left\{\left(\mathcal{W}_{i}, v_{i}\right)\right\}_{i \in I}$ is a $K_{j}$-fusion frame for $\mathcal{H}$, for all $j$, there exist $A, B>0$ such that

$$
A\left\|K_{j}^{*} f\right\|^{2} \leq \sum_{i \in I} v_{i}^{2}\left\|P_{\mathcal{W}_{i}} f\right\|^{2} \leq B\|f\|^{2} .
$$

Then the conclusion follows from the following inequalities:

$$
\frac{A}{\left(\sum_{j}\left|a_{j}\right|\right)^{2}}\left\|\left(\sum_{j=1}^{n} a_{j} K_{j}\right)^{*} f\right\|^{2} \leq \sum_{i \in I} v_{i}^{2}\left\|P \mathcal{W}_{i} f\right\|^{2} \leq B\|f\|^{2},
$$

and

$$
\frac{A}{\prod_{j=2}^{n}\left\|K_{j}^{*}\right\|^{2}}\left\|\left(\prod_{j=1}^{n} K_{j}\right)^{*} f\right\|^{2} \leq A\left\|K_{1}^{*} f\right\|^{2} \leq \sum_{i \in I} v_{i}^{2}\left\|P_{\mathcal{W}_{i}} f\right\|^{2} \leq B\|f\|^{2}
$$

for all $f \in \mathcal{H}$. It may be noted that the trivial case, $K_{j}$ being zero operator, has been omitted.

Suppose $\mathcal{U}$ and $\mathcal{V}$ are two closed subspaces of $\mathcal{H}$ and $P_{\mathcal{U}}, P_{\mathcal{V}}$ are orthogonal projections from $\mathcal{H}$ onto $U, V$, respectively, such that $P_{\mathcal{U}} P_{\mathcal{V}}=P_{\mathcal{V}} P_{\mathcal{U}}$. Then it is well-known that $P_{\mathcal{U}} P_{\mathcal{V}}$ is the orthogonal projection from $\mathcal{H}$ onto $\mathcal{U} \cap \mathcal{V}$. In the following we will discuss when the intersection of $K$-fusion frames is a $K$-fusion frame.

Lemma 4.11. Suppose that $\left\{\mathcal{W}_{i}\right\}_{i \in I},\left\{\mathcal{V}_{i}\right\}_{i \in I}$ are families of closed subspaces of $\mathcal{H}$ and $\left\{w_{i}\right\}_{i \in I},\left\{v_{i}\right\}_{i \in I}$ are families of positive weights. Also suppose that the orthogonal projections $P_{\mathcal{W}_{i}} \& P_{\mathcal{V}_{i}}$ commute for each $i \in I$. If $\left\{\left(\mathcal{W}_{i}, w_{i}\right)\right\}_{i \in I}$ (or $\left.\left\{\left(\mathcal{V}_{i}, v_{i}\right)\right\}_{i \in I}\right)$ is a fusion Bessel sequences in $\mathcal{H}$, then so is $\left\{\left(\mathcal{W}_{i} \cap \mathcal{V}_{i}, w_{i}\right)\right\}_{i \in I}$ (or $\left.\left\{\left(\mathcal{W}_{i} \cap \mathcal{V}_{i}, v_{i}\right)\right\}_{i \in I}\right)$. 
Proof. Suppose $\left\{\left(\mathcal{W}_{i}, w_{i}\right)\right\}_{i \in I}$ is a fusion Bessel sequence. Then for some constant $B>0$, we have for all $f \in \mathcal{H}$

$$
\sum_{i \in I} w_{i}^{2}\left\|P_{\mathcal{W}_{i} \cap \mathcal{V}_{i}} f\right\|^{2}=\sum_{i \in I} w_{i}^{2}\left\|P_{\mathcal{V}_{i}} P_{\mathcal{W}_{i}} f\right\|^{2} \leq \sum_{i \in I} w_{i}^{2}\left\|P_{\mathcal{W}_{i}} f\right\|^{2} \leq B\|f\|^{2}
$$

and hence $\left\{\left(\mathcal{W}_{i} \cap \mathcal{V}_{i}, w_{i}\right)\right\}_{i \in I}$ is a fusion Bessel sequence.

Theorem 4.12. Let $\left\{\left(\mathcal{W}_{i}, w_{i}\right)\right\}_{i \in I}$ be a fusion frame for $\mathcal{H}$ and $\mathcal{V}$ be a closed subspace of $\mathcal{H}$. Also assume $P_{\mathcal{V}}$ commutes with $P_{\mathcal{W}_{i}}$ for each $i \in I$. Then $\left\{\left(\mathcal{W}_{i} \cap\right.\right.$ $\left.\left.\mathcal{V}, w_{i}\right)\right\}_{i \in I}$ will form a $P_{\mathcal{V}}$-fusion frame for $\mathcal{H}$.

Proof. Suppose $\left\{\left(\mathcal{W}_{i}, w_{i}\right)\right\}_{i \in I}$ is a fusion frame for $\mathcal{H}$, then for some constants $A, B>0$ and using Lemma 4.11 we have

$$
A\left\|P_{\mathcal{V}}^{*} f\right\|^{2}=A\left\|P_{\mathcal{V}} f\right\|^{2} \leq \sum_{i \in I} w_{i}^{2}\left\|P_{\mathcal{W}_{i}} P_{\mathcal{V}} f\right\|^{2}=\sum_{i \in I} w_{i}^{2}\left\|P_{\mathcal{W}_{i} \cap \mathcal{V}} f\right\|^{2} \leq B\|f\|^{2},
$$

for all $f \in \mathcal{H}$. Hence $\left\{\left(\mathcal{W}_{i} \cap \mathcal{V}, w_{i}\right)\right\}_{i \in I}$ is a $P_{\mathcal{V}}$-fusion frame for $\mathcal{H}$.

Theorem 4.13. Let $\left\{\left(\mathcal{W}_{i}, w_{i}\right)\right\}_{i \in I}$ be a $K$-fusion frame for $\mathcal{H}$ where $K \in \mathcal{L}(\mathcal{H})$ and $\mathcal{V}$ be a closed subspace of $\mathcal{H}$. Also assume that $P_{\mathcal{V}}$ commutes with $P_{\mathcal{W}_{i}}$ for each $i \in I$ and $P_{\mathcal{V}}^{\dagger}$ commutes with $K^{*}$. Then $\left\{\left(\mathcal{W}_{i} \cap \mathcal{V}, w_{i}\right)\right\}_{i \in I}$ forms a $K$-fusion frame for $R\left(P_{\mathcal{V}}\right)$.

Proof. Since $P_{\mathcal{V}}$ has closed range, $P_{\mathcal{V}}^{\dagger}$ exists. $\left\{\left(\mathcal{W}_{i}, w_{i}\right)\right\}_{i \in I}$ is a $K$-fusion frame for $\mathcal{H}$ implies that there exist positive constants $A, B$ such that

$$
A\left\|K^{*} f\right\|^{2} \leq \sum_{i \in I} w_{i}^{2}\left\|P_{\mathcal{W}_{i}} f\right\|^{2} \leq B\|f\|^{2},
$$

for all $f \in \mathcal{H}$. Therefore using Lemma 44.11, for all $f \in R\left(P_{\mathcal{V}}\right)$, we have $\frac{A}{\left\|P_{\mathcal{V}}^{\dagger}\right\|^{2}}\left\|K^{*} f\right\|^{2}=$ $\frac{A}{\left\|P_{\mathcal{V}}^{\dagger}\right\|^{2}}\left\|K^{*} P_{\mathcal{V}}^{\dagger} P_{\mathcal{V}} f\right\|^{2} \leq A\left\|K^{*} P_{\mathcal{V}} f\right\|^{2} \leq \sum_{i \in I} w_{i}^{2}\left\|P_{\mathcal{W}_{i}} P_{\mathcal{V}} f\right\|^{2}=\sum_{i \in I} w_{i}^{2}\left\|P_{\mathcal{W}_{i} \cap \mathcal{V}} f\right\|^{2} \leq$ $B\|f\|^{2}$. Hence $\left\{\left(\mathcal{W}_{i} \cap \mathcal{V}, w_{i}\right)\right\}_{i \in I}$ is a $K$-fusion frame for $R\left(P_{\mathcal{V}}\right)$.

\section{Conclusion}

In the area of frame theory, the study of atomic subspaces has a great significance to characterize fusion frames with respect to a bounded linear operator, which we have analyzed in Sections 3 \& 4

$K$-fusion frames come naturally when one needs to reconstruct functions from a large data in the range of a bounded linear operator. $K$-fusion frames can be further studied to rich the existing literature of fusion frames and their applications in coding theory, sensor network, etc.

\section{ACKNOWLEDGEMENTS}

The first author acknowledges the financial support of MHRD, Government of India. 


\section{REFERENCES}

[1] P. Casazza and G. Kutyniok, Frames of subspaces, Contemp. Math., AMS 345 (2004), 87-114.

[2] P. Casazza, G. Kutyniok and S. Li, Fusion frames and distributed processing, Appl. Comput. Harmon. Anal., 25 (2008), 114-132.

[3] O. Christensen, An introduction to frames and riesz bases, Birkhäuser, Boston, 2003.

[4] I. Daubechies, A. Grossmann and Y. Meyer, Painless nonorthogonal expansions, J. Math. Phys., 27(5) (1986), 1271-1283.

[5] R. G. Douglas, On majorization, factorization and range inclusion of operators on Hilbert space, Proc. Amer. Math. Soc., 17(2) (1966), 413-415.

[6] R. Duffin and A. C. Schaeffer, A class of nonharmonic Fourier series, Trans. Amer. Math. Soc. 72(2) (1952), 341-366.

[7] Y. C. Eldar, Sampling with arbitrary sampling and reconstruction spaces and oblique dual frame vectors, J. Fourier Anal. Appl., 9(1) (2003), 77-96.

[8] P. Ferreira, Mathematics for multimedia signal processing II: discrete finite frames and signal reconstruction, Signal Processing for Multimedia, J. S. Byrnes, Ed. Amsterdam, The Netherlands: IOS Press (1999), 35-54.

[9] L. Găvruţa, Frames for operators, Appl. Comput. Harmon. Anal., 32(1) (2012), 139-144.

[10] L. Găvruţa, Atomic decompositions for operators in reproducing kernel Hilbert spaces, Math. Rep., 17(67)(3) (2015), 303-314.

[11] R. B. Holmes and V. I. Paulsen, Optimal frames for erasures, Linear Algebra Appl., 377 (2004), 31-51.

[12] S. Jose and K. C. Sivakumar, Chapter 10: Moore-Penrose inverse of perturbed operators on Hilbert spaces, In: Combinatorial matrix theory and generalized inverses of matrices, (R. B. Bapat, S. J. Kirkland and K. M. Prasad, eds.), 119-131, Springer, NewYork, 2013.

[13] A. F. Liu and P. T. Li, K-fusion frames and the corresponding generators for unitary systems, Acta Math. Sin. (Engl. Ser.), 34(5) (2018), 843-854.

[14] G. Ramu and P. Johnson, Frame operators of K-frames, SeMA J. , 73(2) (2016), 171-181.

[15] T. Strohmer and R. W. Heath Jr., Grassmannian frames with applications to coding and communication, Appl. Comput. Harmon. Anal., 14(3) (2003), 257-275.

[16] X. Xiao, Y. Zhu and L. Găvruţa, Some properties of $K$-frames in Hilbert spaces, Results Math., 63(3) (2013), 1243-1255.

Dept. of Mathematics, Nit Meghalaya, Shillong 793003, India

E-mail address: animesh@nitm.ac.in

Dept. of Mathematics, Nit Meghalaya, Shillong 793003, India

E-mail address: saikat.mukherjee@nitm.ac.in 